\title{
SYNCHRO-BETATRON RESONANCES
}

A. Pivinski

Deutsches Elektronen-Synchrotron DESY, Hamburg, Germany

\begin{abstract}
Three different mechanisms are discussed which excite synchro-betatron resonances: the beam-beam interaction at a crossing angle, a dispersion at a cavity and transverse fields with a longitudinal gradient. Experimental results from DORIS and PETRA are shown.
\end{abstract}

\section{INTRODUCTION}

Synchro-betatron resonances or satellite resonances occur when the synchrotron and betatron frequencies satisfy the relation

$$
k Q_{x}+2 Q_{z}+m Q_{S}=n
$$

where $k, l, m$ and $n$ are integers and $Q_{x}, Q_{z}$ and $Q_{s}$ are the betatron and synchrotion frequencies in units of the revolution frequency. These resonances have been a serious problem in storage rings and synchrotrons and will become still more important in larger storage rings. One reason for this is a larger $Q_{s}$ which can go up to 0.2 . Another reason is a larger charge per bunch which increases the strength of most of the satellites.

We will discuss here three different mechanisms which excite synchrobetatron resonances and which limit the performance of storage rings. The first one is the beam-beam interaction at a crossing angle. The second one occurs if there is a dispersion at an accelerating cavity or at any cavitylike structure in the ring. A third mechanism is given by transverse fields which vary with the longitudinal position of a particle in a bunch. The transverse fields can be produced by the accelerating voltage due to asymmetries of the cavities, or by the bunch itself.

We will not discuss here satellite resonances which are excited by the chromaticity of the machine ${ }^{1,2}$, $i . e$. by the energy dependence of the betatron frequencies. The chromaticity in large storage rings is usually compensated with sextupoles so that these resonances will not occur. We will also not consider satellites which are caused by the energy dependence of the amplitude "function ${ }^{14}$ ), since these half integer satellites have not yet been observed.

\section{BEAM-BEAM INTERACTION AT A CROSSING ANGLE}

This effect has limited the luminosity of the storage ring DORIS during its double ring operation lo,ll), and it might become important for the new ep-project HERA ${ }^{17}$ ). Fig. I shows two bunches crossing at an angle of $2 \phi$. 


$$
\sigma_{\text {zef }}=\sqrt{\sigma_{z}^{2}+\phi^{2} \sigma_{s}^{2}}
$$

In the case of a linear coupling one can apply the matrix formalism and calculate the eigenvalues

$$
\lambda=e^{i \mu}
$$

of the revolution matrix which give the stability condition of the motion. A resonance occurs for $Q_{z} \pm Q_{S}=n$ and the phases $\mu$ of the eigenvalues are given by 10,11 )

$$
\mu=\mu_{z, s}+2 \pi \xi \pm 2 \pi \xi \phi \sqrt{\frac{-\alpha_{M} C}{B_{z 0}{ }^{s i n \mu} z}}
$$

with $\mu_{z, s}=2 \pi Q_{Z, S}, \alpha_{M}=$ momentum compaction factor, $C=$ circumference. Here all quadratic terms in $\xi$ are neglected. In the case of an even number $N$ of equidistant interaction points which have crossing angles with alternating signs one has to divide $C$ and $\mu$ by $N$, and Eq. (8) gives the phase advance between two interaction points. Eq. ( 8 ) shows that an instability occurs in the case of a difference resonance with $\sin _{z}$ positive and $Q_{z}$ above an integer.

To get an idea of the strength of the instability we will give the rise times for the ep-project HERA with four horizontal crossings. The HERA parameters are

$$
\begin{gathered}
2 \phi=10 \mathrm{mrad}, C=6451 \mathrm{~m}, \xi_{x}^{+}=0.0007, \xi_{x}^{-}=0.014, \beta_{\times 0}^{+}=7 \mathrm{~m}, \\
B_{X 0}^{-}=5 \mathrm{~m}, Q_{M}^{+}=\alpha_{M}^{-}=0.0005, Q_{S}^{+}=0.012, Q_{S}^{-}=0.054
\end{gathered}
$$

With these parameters one gets a rise time of $100 \mathrm{msec}$ for protons and $9 \mathrm{msec}$ for electrons.

The nonlinear satellite resonances, which occur for large amplitudes, can be investigated with help of approximation methods. Only one result should be given here. One can derive an invariant of motion which is determined by

$$
\hat{z}^{2}+\frac{2 \alpha M B_{z} C}{m 2 \pi Q_{S}}\left(\frac{\Delta \hat{E}}{E}\right)^{2}=\text { const. }
$$

where $\hat{z}$ and $\Delta \hat{E}$ are the amplitudes of the oscillation.

Since $m$ can be positive or negative (Eq.(I)), the betatron and synchrotron amplitude can increase or decrease at the same time or they can exchange their oscillation energy periodically. So an instability occurs again for a difference resonance, whereas in the case of a coupling resonance of horizontal and vertical betatron oscillation the difference resonance is stable and the sum resonance is unstable. The reason for this different behavior is the assumption that the particle energy is above the transition energy. Below the transition energy one has to replace the momentum 


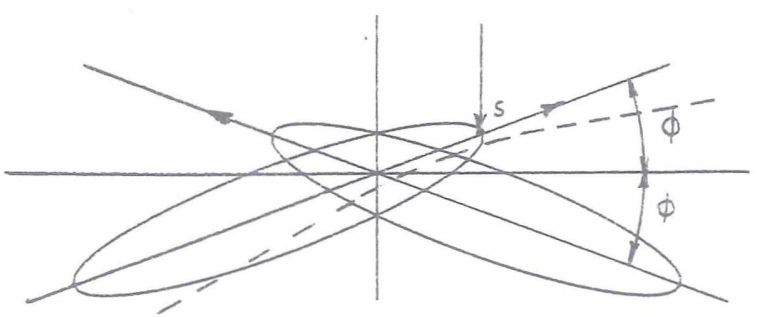

Fig. 1: Drbit distortion due to a crossing angle
We consider a particle which

has a distance $s$ from the center of its own bunch. This particle does not pass through the center of the other bunch and hence gets a vertical kick. The kick has the direction indicated in Fig. 1. if we assume particles with different signs as electrons

and positrons. Because of this kick the axis of the bunch cannot be the closed orbit for the considered particle, but the closed orbit must look like the dotted line. Here we have assumed that the Q-value of the betatron oscillation is above an integer and the dispersion is zero.

The kick, i.e. the change of the vertical betatron angle $z$ ' can be witten in the form

$$
\delta z^{\prime}=f(z+s \phi)
$$

where the function $f$ describes the space charge forces. Eq.(2) shows the influence of the synchrotron oscillation on the betatron oscillation ( $\delta z^{\prime}$ depends on $s$ ). But this is not the whole effect. The synchrotron oscillation is also influenced by the betatron oscillation. This influence can be seen if one remembers that the energy is changed for a particle that crosses the bunch at an angle ${ }^{3}$ ). The energy change is understandable, if one takes into account that the kick or momentum change is vertical. One can then decompose the change of the momentum and obtains a longitudinal component given by

$$
\delta p_{s}=\phi \delta p_{z}
$$

The energy change is then

$$
\frac{\delta E}{E}=\frac{\delta p}{p}=\frac{\delta p_{s}}{p}=\phi \frac{\delta p_{z}}{p}=\phi \delta z^{\prime}=\phi f(z+s \phi)
$$

The energy change depends on the betatron coordinate. So we have the complete coupling which is produced by the crossing angle.

For small oscillation amplitudes the function $f$ may be linearized and one obtains a linear coupling

$$
\begin{aligned}
& \delta z^{\prime}=-\frac{4 \pi \xi}{B_{z 0}}(z+s \phi) \\
& \frac{\delta E}{E}=-\frac{4 \pi \xi \phi}{B_{z 0}}(z+s \phi)
\end{aligned}
$$

with

$$
\xi=\frac{I_{e} N_{b}^{B} z o}{2 \pi \gamma \sigma \sigma_{z e f}\left(\sigma_{x}+\sigma_{z e f}\right)}
$$

$I_{e}=$ electron radius, $N_{b}=$ number of particles per bunch, $B_{z 0}=$ amplitude function at the interaction point, $\gamma=$ particle energy divided by its rest energy, $\sigma_{s}, \sigma_{x}, \sigma_{z}=$ standard deviations for longitudinal, horizontal and vertical bunch dimensions. 
compaction factor $a_{M}$ by $\left(a_{M} \gamma^{2}-1\right) /\left(\gamma^{2}-1\right)$. So below the transition energy $\left.\left(a_{M}\right)^{2}<1\right)$ the satellite resonances show a similar behavior as the coupling resonances of horizontal and vertical betatron oscillations.

For most cases $\hat{z}^{2}$ is smaller than the second term in Eq.(9), and the change of the betatron amplitude is larger than the change of the synchrotron amplitude.

\section{EXPERIMENTAL RESULTS FROM DORIS}

The satellites that were limiting the luminosity of the double storage ring DORIS were mainly those of nonlinear resonances excited by the beambeam interaction at a crossing angle of $24 \mathrm{mrad}(2 \phi)$. Some experiments

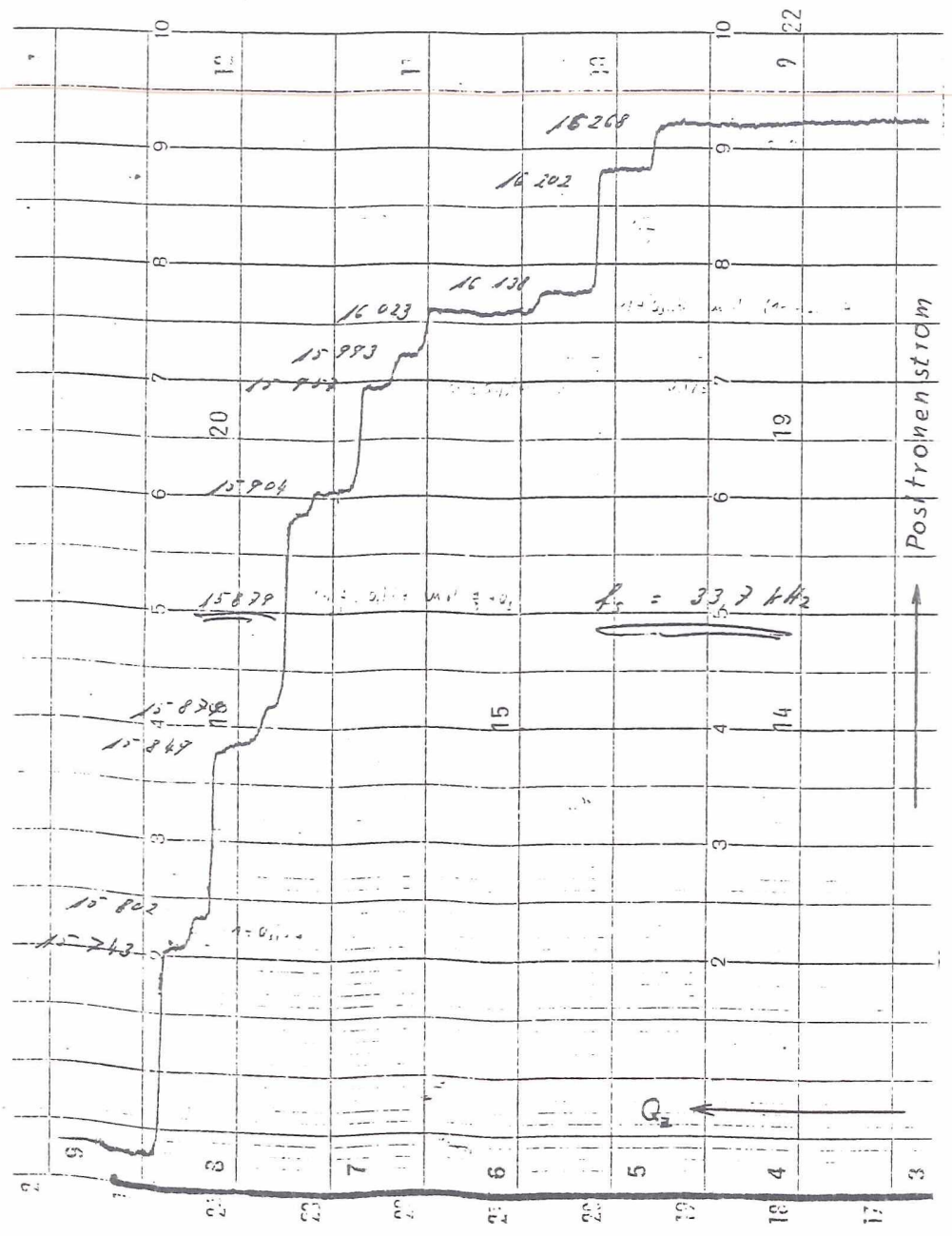

Fig. 2: Beam losses as a function of the vertical betatron frequency 
were made to investigate this limitation. The behavior of weak positron bunches colliding with strong electron bunches was studied. Without decoupling transmitter and without rf-quadrupole a maximum bun current of about $1.3 \mathrm{~mA}$ could be stored in 30 electron bunches. The resulti $\xi$ is 0.009 .

The vertical betatron frequency of the positrons was varied nearly continuously by a computer, whereas the synchrotron frequency was kept constant. The speed of the variation was $1 \mathrm{kHz}$ per $10 \mathrm{sec}$. Simultaneously current of the 30 positron bunches was plotted. The sharp beam losses yie exactly the frequencies of the resonances.

An example of such a measurement is shown in Fig. 2 for one interaction. Because of the very small width of the resonances and because of speed of the variation only those resonances could be observed which lead a life time less than $15 \mathrm{~min}$.

In many measurements with one interaction pointthe following 25 vertical betation resonances were observed:

$$
\begin{aligned}
& 6+3 Q_{S}, 6+4 Q_{S} \\
& 49 / 8,\left(49+Q_{s}\right) / 8,\left(49-2 Q_{s}\right) / 8 \\
& 43 / 7,\left(43 \pm Q_{s}\right) / 7,\left(43-2 Q_{s}\right) / 7 \\
& 37 / 6,\left(37-Q_{s}\right) / 6,\left(37-2 Q_{s}\right) / 6 \\
& 31 / 5,\left(3 I \pm Q_{S}\right) / 5,\left(3 I-2 Q_{s}\right) / 5,\left(31 \pm 3 Q_{s}\right) / 5,\left(31 \pm 4 Q_{s}\right) / 5 \\
& 25 / 4,\left(25-Q_{s}\right) / 4,\left(25-2 Q_{s}\right) / 4 \\
& 28-3 Q_{x}, Q_{x}-1-Q_{s}
\end{aligned}
$$

The life time on a resonance was between a few seconds and $15 \mathrm{~min}$. The width of the resonances was measured in several cases. It was about $5 \times 10^{-4}$.

Although the width of these resonances is very small as compared to the spacing between the resonances, they limit the luminosity of the storage ring DORIS. The limitation is caused by the decoupling transmitter and the rf quadrupole, which are needed to suppress single beam instabilities. The decoupling transmitter gives to different bunches different synchrotron frequencies with a spread of $\Delta Q_{S}= \pm 0.006$. The If quadrupole produces a spread in betatron frequencies of $\Delta Q_{z}= \pm 0.005$. Thus with these operating conditions there are always some bunches on a resonance.

\section{DISPERSION AT A CAVITY}

Synchro-betatron resonances which are excited by a dispersion in the cavities were first observed in NINA ${ }^{4}$ ) and several studies were made to understand this mechanis ${ }^{5-9,13}$ ). The physical model can be described as follows. 
For each particle with an arbitrary energy deviation exists a close orbit that can be described as the product of the dispersion D times th relative momentum deviation or approximately, the relative energy deviation:

$$
x_{c .0 .}(l)=D_{x}(l) \frac{\Delta p}{p} \approx D_{x}(l) \frac{\Delta E}{E}
$$

Around this closed orbit the particles perform betatro oscillations: The dispersion can be horizontal or vertical and we ca consider horizontal or vertical betatron oscillations. In the cavity th energy is changed and therefore the closed orbit is shifted (Eq.(10)) Since the total coordinate $x$ cannot be changed in such a short intervall the betatron coordinate is changed:

$$
\delta x=-D_{x} \frac{\delta E}{E}=-D_{x} \frac{e U}{E} \cos \psi_{s} \cdot \psi
$$

with $e=$ elementary charge, $U=$ cavity voltage, $\psi_{s}=$ synchronous phase, $\psi=$ phase angle of the synchrotron oscillation

In a similar way one obtains

$$
\delta x^{\prime}=-D_{x}^{\prime} \quad \frac{\delta E}{E}=-D_{x}^{\prime} \frac{e U}{E} \cos \psi_{s} \cdot \psi
$$

We take into account only that part of the energy change whic varies with the synchrotron frequency. The other constant part whic replaces the radiation losses does not play a role for a resonance.

Eqs.(11) and (12) show that there is a linear coupling from the synchrotron oscillation to the betatron oscillation. But the synchrotro oscillation is also influenced by the betatron motion. The synchrotrol oscillation can be described in terms of the relative energy deviation $\Delta E /$ and the longitudinal position s. The longitudinal position is changed $i$ the curved sections of the machine. The change of s per revolution is give by

$$
\delta s=\oint \frac{l}{\rho}\left(x+D_{x} \frac{\Delta E}{E}\right) d \ell
$$

$\rho$ is the radius of curvature and $d l$ is an element of the length of thi design orbit. Evaluation of the integral gives

$$
\delta s=A_{1} x^{\prime}+A_{2} x^{\prime}-\alpha_{M} C \frac{\Delta E}{E}
$$

The constants $A_{1}$ and $A_{2}$ are given by

$$
\begin{aligned}
& A_{1}=-\frac{1}{B_{x}}\left(D_{x} \sin \mu_{x}-F_{x}\left(1-\cos \mu_{x}\right)+A_{2} \beta_{x}^{\prime} / 2\right) \\
& A_{2}=-D_{x}\left(1-\cos \mu_{x}\right)-F_{x} \sin \mu_{x}
\end{aligned}
$$

$\mu_{x}$ is the betatron phase advance per revolution.

$F_{x}$ is a combination of the derivatives of the dispersion $D_{x}$ and the ampli. tude function $\beta_{x}: \quad F_{x}=D_{x}^{\prime} \beta_{x}-D_{x}^{\beta} x_{x}^{1 / 2}$

$D_{x}$ and $F_{x}$ refer to the beginning or to the end of the revolution. 
$x$ and $x$ ' are the betatron coordinate and the betatron angle at the beginning of the revolution.

Eq.(14) shows a linear coupling from the betatron oscillation to the synchrotron oscillation which is caused by the path lengthening due to the betatron oscillation. So the whole coupling is linear. If we assume linear betatron and synchrotron oscillations we can find an exact solution for the coupled motion. The phases $\mu$ of the eigenvalues $\lambda(E q \cdot(7)$ ) of the revolution matrix are then given by ${ }^{9}$ )

$$
\mu=\mu_{x, s} \pm\left(1-\cos \mu_{x}\right) \sqrt{\frac{-\left(D_{x}^{2}+F_{x}^{2}\right)}{\sin \mu_{x} \alpha_{M}{ }^{C} \beta_{x}}}
$$

This expression shows again that the phase $\mu$ becomes complex, if sin ${ }_{x}$ is positive, i.e. an instability occurs for difference resonances.

Since the coupling is linear satellites of the form $Q_{x}+m Q_{S}=n$ with $|m|>I$ can be excited only by nonlinear synchrotron oscillations (Bessel side bands should be very small). A nonlinearity does not only come from the natural synchrotron potential but also from potential distortions which are caused by large bunch currents.

Although the design value of the vertical dispersion is zero in most machines a vertical dispersion can be produced by dipole errors and correcting coils ${ }^{6}$ ). Such a dispersion can become very large in large storage rings. Satellite resonances can then be excited, if a dispersion is produced at the cavities. Satellite resonances are not excited at the position of the dipole error or correcting coil, since the emittance of the betatron oscillation is changed only during the energy variation. So the position of the cavities plays an important role for the excitation ${ }^{9}$ ).

\section{TRANSVERSE FIELDS WITH A LONGITUDINAL GRADIENT}

Transverse fields which vary with the longitudinal position of a particle in a bunch can be caused by the accelerating voltage due to asymmetries in the cavities, for instance, due to an angle between the cavity axis and the closed orbit. This case was investigated in ${ }^{12}$ ). Transverse fields can also be excited by the bunch itself, either in the cavities due to asymmetries or off-center passage, or in any cavity-like structure of the vacuum chamber. This case was investigated in ${ }^{15}$ ).

The vertical kick of a particle produced by transverse fields is given by

$$
\delta z^{\prime}=\frac{e}{p} \int\left(E_{z}+v B_{x}\right) d t
$$

where $E_{z}$ and $B_{x}$ are the electric and magnetic field and $v$ is the particle velocity. The integral is taken along the path of the particle between two limits where the fields vanish. The region of the fields is assumed to be short as compared to the wave length of the betatron oscillation.

We consider that part of $\delta z^{\prime}$ which varies linearly with the longitudinal position $s$ of a particle in the bunch: 


$$
\begin{gathered}
-8- \\
\delta z^{\prime}=s \cdot \frac{e}{p} \int\left(\frac{\partial E}{\partial s}+v \frac{\partial B}{\partial s}\right) d t
\end{gathered}
$$

With Maxuell's equation

$$
\frac{\partial E_{z}}{\partial s}-\frac{\partial E_{s}}{\partial z}=\frac{\partial B_{x}}{\partial t}
$$

one obtains

$$
\delta z^{\prime}=s \cdot \frac{e}{p}\left(\int \frac{\partial E_{s}}{\partial z} d t+\left.B_{x}\right|_{t_{1}} ^{t_{2}}\right)=A s
$$

with

$$
A=\frac{e}{p} \int \frac{\partial E_{s}}{\partial z} d t
$$

If the transverse fields have a longitudinal gradient the longitudinal field must have a transverse gradient, and that means that the betatron oscillation influences the synchrotron oscillation. That part of the energy change which varies linearly with the vertical position of a particle in the bunch is given by

$$
\frac{\delta E}{E}=z \cdot \frac{e}{E} \int \frac{\partial E s}{\partial z} v d t=\frac{v^{2}}{c^{2}} A \cdot z \approx A \cdot z
$$

Eqs. (19) and (21) describe a linear coupling which can be investigated again using the matrix formalism.

On the linear resonance $Q_{z} \pm Q_{S}=n$ the phases of the eigenvalues of the revolution matrix can be written as

$$
\mu=\mu_{z, s} \pm \frac{A}{2} \sqrt{-\frac{B_{z c} \alpha_{M} C}{\sin \mu_{z}}}
$$

where $B_{z c}$ is the amplitude function at the cavity. Eq.(22) shows again that the oscillation amplitudes increase exponentially on a difference resonance.

Also in the case of transverse fields an invariant for the synchrotron and betatron amplitudes can be derived (Eq. (9) with $\ell=1$ ) which shows that usually the change of the betatron amplitude is larger than the change of the synchrotron amplitude. One can then assume a constant synchrotron amplitude and calculate the grouth time of the betatron amplitude which is given by

$$
\frac{d \hat{z}}{d t}=\frac{A}{2} f_{0} \beta_{z c} \hat{s}
$$

The same growth is obtained also from the beat on a sum resonance.

Comparison of Eqs.(11), (12) and (19) shows that the increase of betatron oscillation depends in the same way on the synchrotron oscillation, namely on $\psi$ and $s \sim \psi$. The two mechanisms, caused by a dispersion and by transverse fields, can at least for slowly changing synchrotron amplitude compensate each other. This is also possible for higher harmonics of the synchrotron frequency produced by nonlinearities. Then the compensation depends on $\hat{s}$ and on the bunch current.

Harmonics of the synchrotron frequency can be excited by different nonlinearities. A strong nonlinear coupling occurs if the transverse fields are induced by the bunch. Harmonics also occur due to the nonlinearity of the synchrotion oscillation and due to Bessel side bands. 


\section{EXPERIMENTAL RESULTS FROM PETRA}

In the neighbourhood of the usual working point the following synchro-betatron resonances cause beam loss in the storage ring PETRA:

$$
\begin{array}{lll}
Q_{x}-m Q_{S}=25 & \text { with } & m=2,3,4 \\
Q_{z}-m Q_{s}=23 & \text { with } & m=3,4,5
\end{array}
$$

$Q_{s}$ varies from 0.05 to 0.14 . The satellite of the difference resonance $Q_{x}-Q_{z}+Q_{s}=2$ is also observed but is not as strong as the other satellites.

The resonances have the following properties:

a) Increase of beam dimensions and reduction of life time The transverse bunch dimensions can be enlarged by several standard deviations and the life time can be reduced to a few seconds.

b) Strong dependence on single bunch current Most of the resonances show a strong dependence on the single bunch current (but not on the total beam current). With decreasing current the strength of the satellites goes to zero and the current goes asymptotically to a residual current.

c) Constant short life time on $Q_{x, z}-2 Q_{s}=n_{x, z}$ These satellites have a short life time (a few seconds) even for very small currents, and the residual current is zero. This is observed in a low $\beta$ optics but is not found in an injection optics.

d) Strong dependence on orbit position

All satellites show a strong dependence on orbit position, but the orbit with minimum satellite strength usually differs from the orbit obtained by automatic orbit correction.

e) Energy dependence

All current dependent satellites are weaker at higher energies, but for the satellite $Q_{x, z}-2 Q_{s}=n_{x, z}$ the residual current is zero also at $18 \mathrm{GeV}$.

f) Dependence on the number of cavities

The strength of the satellites with 32 and with 60 cavities was not significantly different. With 4 or 16 cavities the satellites did not disturb the operation, but at that time $Q_{S}$ was smaller.

g) No dependence on chromaticity and feedback.

We found that two types of orbit bumps had a strong influence on the strength of the satellites. Bumps of the first type are in the cavity sections, and bumps of the second type are in the interaction regions, having large amplitudes in the large quadrupoles and therefore producing a large dispersion in the whole ring. 
Fig. 3 shows a compensation of a horizontal satellite with these bumps. The residual current on the resonance is plotted as a function of the bump amplitudes. The first bump in a cavity section gave a weak maximum. The bump was left at the maximum and another bump was varied which had a phase advance of $90^{\circ}$ with respect to the first bump. The bump was again left at the maximum and two bumps vere varied which produce two orthogonal dispersions. After optimizing the last bump which delivered a sharp maximum also the first bump showed a similarly sharp maximum. The width of the maximum corresponds to only about $1 \mathrm{~mm}$ orbit displacement and about $10 \mathrm{~cm}$ dispersion.

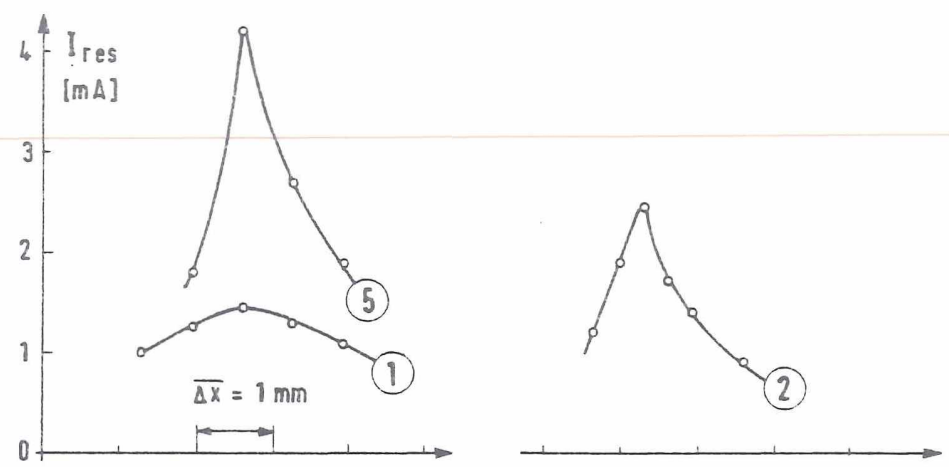

Residual current as a function of two orthogonal bumps in the cavity section
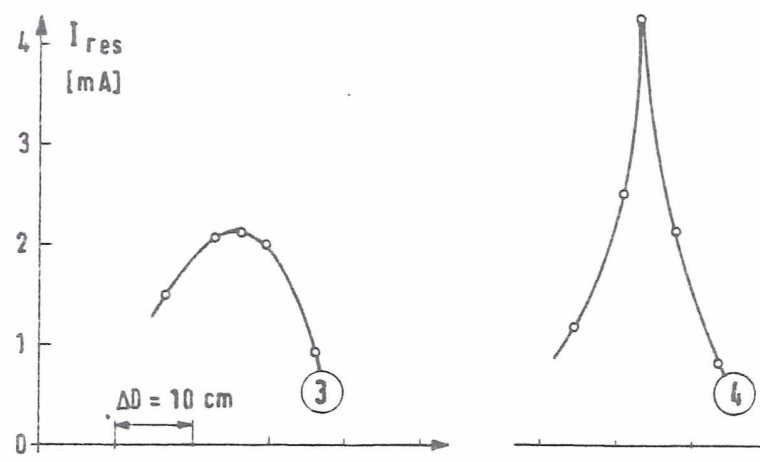

Residual current as a function of two bumps which produce two orthogonal dispersions

Fig. 3: Compensation of the horizontal satelite $Q_{x}=25+3 Q_{S}$ with $Q_{S}=0.058$ 
Fig. 4 shows for the satellite $Q_{X}-2 Q_{S}=25$ that the optimization of the residual current and the optimization of the life time of a current of $0.1 \mathrm{~mA}$ gave two different optimum bump amplitudes. For the same bump amplitude where a current of more than $1 \mathrm{~mA}$ had a life time of more than I h a current of $0.1 \mathrm{~mA}$ had a life time of less than I min. This effect can be explained by the assumption that the satellite is excited by two different mechanisms which can compensate each other and which have a different current dependence.

The measurements have shown ${ }^{16}$ ) that the satellites in PETRA are excited by dispersions in the cavities and by transverse fields in the cavities which have a short decay time (low Q). In principle all the satellites can be compensated with orbit bumps. But due to the very sensitive dependence on the orbit it seems impossible to maintain the compensation during energy ramping. Therefore one has to control the synchrotron and betatron frequencies and stay between the resonances. This can become difficult if a large synchrotron frequency is needed to avoid instabilities at injection energy, whereas the rf limitation at higher energies requires a smaller synchrotron frequency. Then one must cross a satellite and this can limit the current.

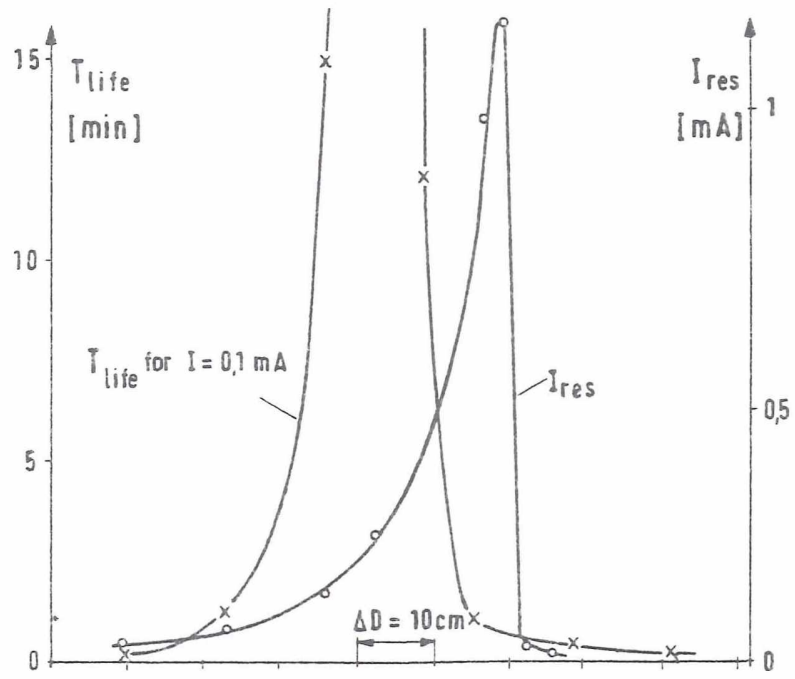

Fig. 4: Life time of a current of $0.1 \mathrm{~mA}$ and residual current on the satellite $Q_{x}-2 Q_{s}=25$ as a function of a bump producing a dispersion 


\section{REFERENCES}

1) Yu. F. Orlov, Sov. Phys. JETP, Vol.5, No.1, 45 (1957)

2) K.W. Robinson, CEA - 54 (1958)

3) J.E. Augustin, Note Interne 35-69 (1969)

4) M.C. Crowley-Milling, I.I. Rabinowitz, 1971 Particle Accelerator Conf., IEEE Trans. on Nuclear Science, NS-18, No.3, 1052, (1971)

5) M.H.R. Donald, RHEL/M/Nim 18, (1973)

6) H.G. Herevard, Interner Bericht DESY PET-75/2 (1975)

7) SPEAR Group, 1975 Particle Accelerator Conference, IEEE Trans.on Nuclear Science, NS-22, 1366 (1975)

8) A.W. Chao, E. Keil, A.S. King, M.J. Lee, P.L. Morton, J.M. Peterson, SPEAR-187, Aug. 1975

9) A. Piwinski, A. Wrulich, DESY 76/07 (1976)

10) A. Piwinski, DESY $77 / 18$ (1977)

11) A. Piwinski, 1977 Particle Accelerator Conference, IEEE Trans. on Nuclear Science, NS-24, No.3, 1408 (1977)

12) N.A. Vinokurov et al., X International Conference on High Energy Accel., II, $254(1877)$

13) A.W. Chao, A. Piwinski, Internal Report DESY PET-77/09 (1977)

14) A. Wrulich, Interner Bericht DESY PET-77/03 (1977)

15) R.M. Sundelin, 1979 Particle Accelerator Conference, IEEE Trans. V.NS -26, No.3, 3604 (1979)

16) D. Degèle et al., DESY $80 / 10$ (1980)

17) Study on the proton-electron storage ring project HERA, ECFA $80 / 42$, 